\title{
AVALIAÇÃO DA MEMÓRIA DE TRABALHO EM CRIANÇAS COM TRANSTORNO DO DÉFICIT DE ATENÇÃO E HIPERATIVIDADE' ${ }^{1}$
}

\section{Lucinete de Freitas Messina Klaus Bruno Tiedemann}

Resumo: Esse projeto investigou as habilidades cognitivas da Memória de Trabalho de crianças e adolescentes com o "Transtorno do Déficit de Atenção e Hiperatividade" (TDAH). Foi aplicado teste TIHC - Teste Infantil de Habilidades Cognitivas informatizado, avaliando principalmente cinco habilidades cognitivas Raciocínio Indutivas (RI), Memória de Armazenamento Auditiva (MAA) e Visual e Memória de Trabalho Auditiva (MTA) e Visual (MTV) usando o modelo de Cattell-Horn-Carroll.O teste foi aplicado em 62 sujeitos sendo 32 com TDAH do Hospital das ClínicasSEPIA-USP e 30 sujeitos controle "normal" do Ensino Fundamental da rede municipal de $1^{1}$ à $8^{\text {a }}$ séries com idades entre 7 e 15 anos, de ambos os sexos. As análises executadas indicaram a existência de diferenças em diversos aspectos relacionados à memória nos tipos peculiares de crianças com TDAH.Verificou-se que as crianças com TDAH obtiveram bom desempenho nas provas de memória visual em detrimento as provas de memória auditiva. A correlação dos resultados do TIHC verificara que o tempo de reação da prova de memória de armazenamento visual manteve uma correlação significativa com a prova de cálculo.

Palavras-chave: Memória. Déficit de Atenção. Hiperatividade. TDAH. Neuropsicologia.

1 Pesquisa de mestrado do Núcleo de Neurociência e Comportamento do Instituto de Psicologia da Universidade de São Paulo e Departamento de Psiquiatria da Faculdade de Medicina da Universidade de São Paulo. Protocolo de pesquisa n.030/04, aprovado pela comissão de ética para análise de projetos de pesquisa no dia 25/03/2004 do Hospital das Clínicas da Faculdade de Medicina da Universidade de São Paulo. 


\section{Introdução}

Respeitando a nomenclatura brasileira, que prefere utilizar o termo transtorno em vez de distúrbio, será utilizada neste projeto a nomenclatura "Transtorno do Déficit de Atenção e Hiperatividade" para "Distúrbio com Déficit de Atenção e Hiperatividade", segundo a conceitualização estabelecida de pelo DSM-IV (American Psychiatry Association [APA], 1994). Esse transtorno descreve as crianças e os adultos que são incapazes de modularem a atenção, controlar a impulsividade e ausência de uma atividade motora apropriada. A formalização atual do Diagnostic and Statistical Manual of Mental Disorders - Fourth Edition (APA, 1994), propõe a caracterização da sintomatologia em três subtipos específicos: subtipo predominantemente desatento, subtipo hiperativo ou impulsivo e subtipo combinado. Além dessa proposta, o DSM-IV inclui uma classificação não específica aos distúrbios que não satisfazem os critérios para a tríade do TDAH. Para auxílio no diagnóstico, o DSM-IV lista 18 sintomas que definem as duas principais sintomatologias do TDAH: predominantemente desatento (nove sintomas) ou hiperativo-impulviso (com seis sintomas de hiperatividade e três sintomas relacionados à impulsividade).

As diferenças existentes entre os três subtipos de TDAH no que diz respeito às regiões neuroanatômicas envolvidas são a forma sem hiperatividade (predominantemente desatenta) que parece envolver áreas corticais associativas posteriores ou alças subcorticais incluindo provavelmente o hipocampo, e a forma com hiperatividade (predominantemente hiperativa ou mista) que envolveria vias pré-frontal-límbicas incluindo os circuitos fronto-estriatais (Barkley, Grodzinsky, \& DuPaul, 1992; Castellanos, 1997; Castellanos et al., 1997; Castellanos, Ducharme, Walter, Israel, Krain, Pavlovsky, 2000). Os subtipos também se diferenciariam quanto aos perfis neuropsicológicos apresentados pelo paciente, que poderia contribuir também para uma diferença no desenvolvimento de outros transtornos mentais ao longo do tempo. Enquanto o subtipo sem hiperatividade associa os problemas envolvendo a atenção seletiva e a velocidade de processamento de informações, o tipo com hiperatividade associa as dificuldades na sustentação da atenção ao longo do tempo, com maior vulnerabilidade à distração. Assim, enquanto os portadores de forma sem hiperatividade apresentam desempenhos piores nos testes de destreza vísuo-motora, de velocidade de processamento e de recuperação mnêmica verbal, aqueles com a forma com hiperatividade, em suas performances nestas capacidades, não diferem dos sujeitos controles normais (Sonuga-Barkley, Taylor, \& Sembi, 1992). O desempenho acadêmico de portadores da forma sem hiperatividade tende a ser pior, se comparado aos subtipos que apresentam a hiperatividade e consequentemente aos problemas comportamentais aos quais a hiperatividade pode estar associada (Babinski, Hartsough, \& Lambert, 1999; Rohde, 1997; Strauss \& Alphal Boekamp, 1992). 
Outro critério importante para o espectro comportamental do transtorno é a idade. Antes dos sete anos, os primeiros sintomas devem ser observados em pelo menos dois contextos sociais (ex.: casa e escola, casa e lugar de trabalho etc), realçando um prejuízo essencial dos sintomas no ambiente social, ocupacional ou de aprendizagem. Uma compreensão dessas características faz necessário o estudo das variáveis idade e desenvolvimento do indivíduo. Quando há outras desordens de desenvolvimento, esquizofrenia e outras psicoses infantis, exclui-se o diagnóstico de TDAH.

A prevalência do TDAH é estimada em 3\% a 5\%, segundo a Associação Americana de Psiquiatria (APA, 1994). É o diagnóstico mais comum na infância em diferentes sociedades, incluindo o Brasil (Barbosa, Barbosa, \& Nascimento, 1999a, 1999b; Barbosa, Gouveia, \& Barbosa, 1993; Rohde et al., 1998). Esses valores podem variar em função do modo como é feito o diagnóstico, utilizando-se critérios da Associação Psiquiátrica Americana, o Diagnostic and Statistical Manual IV, a CID-10, por entrevista clínica estruturada ou por critérios neuropsicológicos. A prevalência do TDAH no sexo masculino, comparada com o sexo feminino, é desproporcional, com taxas de quatro vezes mais nos meninos do que nas meninas. À medida que o indivíduo cresce, ocorrem também taxas crescentes de co-morbidade, ou seja, outros transtornos mentais (Arnold et al., 1997; Kendall \& Chansky, 1991; Kuntsi, Oosterlaan, \& Stevenso, 2001).

Rohde et al. (1998) ressalta que a pesquisa clínica de co-morbidades associadas ao TDAH é fundamental. A prevalência elevada das co-morbidades aponta para decisões terapêuticas distintas. Assim, os transtornos mentais que mais se apresentam associados com o TDAH são: transtorno de conduta (40\%), depressão (21\%), transtorno de ansiedade (18\%), transtorno bipolar (12\%) e transtorno de aprendizagem (10\%). Correia e Rohde (1998) verificaram em seus estudos que $50 \%$ dos pacientes com transtorno de Tourette e tiques apresentam TDAH. Dentre os adolescentes com déficit de atenção há uma maior incidência de abuso de drogas principalmente quando adultos. Esse fato provavelmente é dependente da ligação com transtornos de conduta, uma vez que se sabe que há uma relação entre distúrbios de conduta na infância e abuso de drogas no adulto.

A criança ou adolescente com TDAH não é um diagnóstico fácil de ser dado, há uma grande dificuldade em diagnosticar esses indivíduos no meio clínico. Além da dificuldade de classificação e critério de diagnóstico, existe uma outra que também é fundamental para a avaliação da criança com TDAH, é a inexistência de padrões ou normas que estabeleçam a variação do nível da atividade motora e nível de atenção/concentração que pode ser considerado para o diagnóstico da hiperatividade ou do déficit de atenção.

$\mathrm{O}$ TDAH, em sua etiologia, pode ocorrer como resultado de bases multifatoriais, nos fatores genéticos, biológicos e neuropsicológicos. $\mathrm{Na}$ genética, a predisposição é acima de 50\% no TDAH (Barbosa, Gouveia, \& Barbosa, 2000). A deficiência biológica recebe suporte dos estudos com 
imagens cerebrais, em que o TDAH é basicamente uma disfunção geneticamente herdada do córtex pré-frontal, devido, em parte, a uma deficiência do neurotransmissor dopamina. Através dos estudos neuropsicológicos, também sugerem que o TDHA está associado a alterações do córtex préfrontal e de suas projeções a estruturas subcorticais, o que caracteriza esse transtorno por frequentes níveis de desatenção, impulsividade, hiperatividade, desorganização e inabilidade social, envolvendo um déficit do sistema inibitório ou as funções executivas da memória de trabalho do TDAH (Baddeley, 1996; Baddeley, Logie, Bressi, Della Salla, \& Spinnler, 1986, citados por Gathercole \& Baddeley, 1993).Vários pesquisadores têm mostrado que as características principais do Transtorno do Déficit de Atenção com Hiperatividade (TDAH) estão associadas a uma dificuldade para inibir ou frear impulsos. Partindo desses achados, Dr. Barkley (1997) afirmou que essa dificuldade na inibição dos impulsos não permite que alguns dos processos psicológicos, como as funções executivas, possam operar eficientemente.

Essas disfunções comportamentais e cognitivas no TDAH podem interferir diretamente na vida acadêmica dessas crianças, com consequências drásticas ao desenvolvimento global (Meili, 1985; Posner \& Cohen, 1984; Pennington \& Ozonoff, 1996; Swanson \& Posner, 1991). Em muitos casos, a etiologia do TDAH permanece obscura, e há uma emergência na necessidade de mais pesquisas para auxiliar na prevenção e cura do transtorno.

Apesar de não haver uma etiologia totalmente definida para oTDAH, parece haver atualmente um consenso entre os pesquisadores, apontando para a área frontal e suas projeções. Em concordância a isso, a literatura indica que o sistema noradrenérgico central parece estar desregulado no TDAH, passando a não modular eficientemente o sistema de atenção do córtex posterior aos estímulos externos (Balthazor, Wagner, \& Pehan, 1991; Cohen et al., 2000; Kuntsi et al., 2001). Os trabalhos podem ser reunidos em dois grandes grupos: um que aponta para o déficit funcional de certos neurotransmissores, e outro que dá ênfase ao déficit funcional do lobo frontal, mais precisamente do córtex pré-frontal.

Em uma revisão na literatura de 2002 a 2004 foram encontrados 1.774 artigos de TDAH (fontes: MEDLINE e PsycINFO), verificou-se que as pesquisas com TDAH estão assumindo grandes proporções no território brasileiro, observa-se que há poucas contribuições que relacionam esse transtorno com a memória de trabalho na população em geral. Diante disso, visando dar uma contribuição nos estudos da interação do TDAH e da memória de trabalho, nesse estudo apresenta-se a utilização de cinco subtestes de avaliação das capacidades específicas da memória de trabalho segundo o Modelo C-H-C. Esse modelo contribuiu para dar consistência e credibilidade a uma das teorias cognitivas mais aceitas atualmente sobre "inteligência" humana. Após uma revisão das pesquisas psicométricas dos últimos 60 anos, Carroll (1993), junto com Cattell e Horn, integram suas teorias para compreensão da inteligência humana, sendo considerado uma 
evolução das idéias contidas no modelo fatorial da estruturas das habilidades cognitivas humanas (Pasquali, 2001; Primi, 2002).

Em neurociência ocorre atualmente uma mudança rápida e gradual, porém inexorável, de paradigma. Os modelos teóricos mais tradicionais, baseados na teoria do processamento de informação e em modelos da inteligência artificial clássica estão sendo substituídos por concepções derivadas dos modelos de redes neurais e de construtos da dinâmica nãolinear (Del Nero, 1997; Teixeira, 1998). Essas transformações teóricas implicam também em profundas modificações no modo como os construtos são medidos, seja para fins de pesquisa, seja para aplicações clínicas, como no contexto da avaliação neuropsicológica. A memória de trabalho é um construto que não foge a essa regra, ilustrando tanto as vantagens quanto às dificuldades de pensar dinamicamente em neuropsicologia. Segundo a formulação original de Baddeley (1996a, 1996b), a memória de curta duração não se constitui apenas em um reservatório temporário de informação, mas cumpre também um papel ativo, executivo no seu processamento. Baddeley concebeu o modelo canônico de organização da memória de trabalho como um sistema tripartite de armazenamento constituído de um executivo central e dois sistemas escravos: uma alça fonológica, relacionada à representação e recitação do material verbal, e um sistema tampão vísuo-espacial, o equivalente imagético da alça fonológica. As propriedades dos dois sistemas escravos estão relativamente bem caracterizadas, inclusive o seu papel no desenvolvimento normal e patológico e o seu funcionamento após lesões em diversas áreas cerebrais (Baddeley, 1996a, 1996b, Baddeley \& Hitch, 1974, citados por Gathercole \& Baddeley, 1993).

A alça fonológica é responsável pela manutenção de material verbal, funciona através de código fonológico. Seu armazenamento é de capacidade restrita, devido ao material se "deteriorar" rapidamente com o tempo, para que não haja prejuízo do material verbal, a alça fonológica comporta em si dois sistemas complementares, um destinado à estocagem fonológica passiva, e outro relativo aos processos de ensaios articulatórios. No modelo de Baddeley (1998) a alça fonológica é responsável pela retenção de curto prazo de uma informação verbal (Baddeley, 1998; Gathercole \& Baddeley, 1993).

A alça vísuo-espacial considera-se que seja análoga à alça fonológica por também manter informações que podem ser ativamente ensaiadas, porém de natureza vísuo-espacial. A exemplo da alça fonológica, este módulo também comporta dois sistemas complementares, um responsável pela manutenção passiva de material visual, outro pela manipulação de material espacial. A competência desse sistema está em processar e armazenar informações visuais e espaciais, bem como o material verbal codificado na forma de imagem. Este componente vísuo-espacial da memória de trabalho está envolvido tanto na retenção da informação visual quanto 
na geração e manutenção de imagens codificadas em forma de figuras ou letras (Baddeley, 1998; Farner \& O'donnell, 2000).

Dos três componentes que constituem a memória de trabalho, o mais importante é o executivo central, ou sistema de controle de atenção, servido por dois sistemas subordinados: a alça fonológica e alça vísuo-espacial.

O executivo central é o principal componente da teoria, e tem como funções o raciocínio, a tomada de decisões, o planejamento de estratégias e o controle do comportamento por meio da integração das informações dos sistemas subordinados. Sua operação envolve a aprendizagem e aplicação de regras contingentes, raciocínio abstrato, e manutenção da atenção e concentração e seu funcionamento são estreitamente relacionados às áreas pré-frontais (Baddeley, 1996). As tarefas cognitivas que envolvem o executivo central incluem: aritmética mental (Adams \& Gathercole, 1996), recordar listas prolongadas de dígitos, raciocínio lógico (Baddeley \& Hitch, 1974, citados por Gathercole, \& Baddeley, 1993), verificação semântica (Baddeley; Lewis, Eldridge, \& Thomson, 1984, citados por Gathercole, \& Baddeley, 1993), e a lembrança de eventos da memória de longo prazo (Adams \& Gathercole, 1996).

Como esse trabalho trata de fatores específicos das capacidades cognitivas da memória de trabalho e de armazenamento tanto visual quanto auditivo, procurou-se enfatizar quais seriam as possíveis dificuldades que poderiam decorrer de baixos níveis nessas capacidades. Em relação ao raciocínio indutivo, dificuldades detectadas nessa capacidade implicariam em dificuldade para generalizar regras, formar conceitos e observar implicações, dificuldade em reorganização, transformação e generalização das informações bem como dificuldade em raciocinar com conteúdos abstratos como a matemática. Já em relação à memória de trabalho e de armazenamento, as dificuldades incidirão sobre a manutenção da atividade de informações recém-adquiridas tanto na modalidade auditiva quanto visual. Dificuldades de memória de trabalho afetam grande parte do processamento de informação, uma vez que a memória é uma estrutura mediadora das informações.

Partindo-se dessas informações buscaram-se levantar hipóteses sobre a quais dificuldades estas capacidades poderiam estar associadas. Para isso utilizou-se o esquema de Pennington (1997) para definição dos distúrbios da aprendizagem. No quadro 5 são apresentadas as relações entre as funções cerebrais (primeira coluna) e seus possíveis distúrbios (terceira coluna) segundo Pennington (1997). Na segunda coluna acrescentamos os fatores cognitivos segundo o modelo $\mathrm{CHC}$ que se hipotetizou estarem relacionados a essas funções. Cabe salientar que as relações são baseadas em análises teóricas necessitando investigação empírica para que se possam confirmar estas relações. Pode-se observar que o raciocínio indutivo (inteligência fluida) e a memória de trabalho podem estar associados às funções executivas, de processamento fonológico e à cognição espacial e 
consequentemente ao distúrbio do déficit de atenção, à dislexia e a distúrbios específicos da matemática.

Quadro: Funções cerebrais e seus distúrbios de acordo com Penington (1997) e sua relação com as habilidades cognitivas do modelo CHC.

\begin{tabular}{|c|c|c|c|}
\hline $\begin{array}{c}\text { Função } \\
\text { executiva }\end{array}$ & $\begin{array}{l}\text { Processo } \\
\text { associado }\end{array}$ & $\begin{array}{c}\text { Habilidades sociais e } \\
\text { psicológicas }\end{array}$ & $\begin{array}{c}\text { Provável } \\
\text { localização } \\
\text { cerebral }\end{array}$ \\
\hline $\begin{array}{l}\text { Inibição de } \\
\text { resposta }\end{array}$ & $\begin{array}{l}\text { Inibição de resposta; } \\
\text { Interrupção } \\
\text { de respostas } \\
\text { contínuas; Controle } \\
\text { de interferências } \\
\text { (distração). }\end{array}$ & $\begin{array}{l}\text { Controle impulsivo e auto- } \\
\text { regulação; Retardo na } \\
\text { gratificação; Regulação do } \\
\text { nível de atividade de acordo } \\
\text { com a demanda da tarefa. }\end{array}$ & $\begin{array}{l}\text { Pré-frontal orbital } \\
\text { e estriado }\end{array}$ \\
\hline $\begin{array}{l}\text { Memória de } \\
\text { trabalho não } \\
\text { verbal }\end{array}$ & $\begin{array}{l}\text { Função retrospectiva } \\
\text { Função prospectiva }\end{array}$ & $\begin{array}{l}\text { Manter eventos na mente; } \\
\text { Imitação/aprendizado } \\
\text { vicariante; Senso do passado; } \\
\text { Senso do futuro; Senso } \\
\text { do tempo; Organização } \\
\text { temporal do comportamento. }\end{array}$ & $\begin{array}{l}\text { Pré-frontal } \\
\text { dorsolateral } \\
\text { (direito maior } \\
\text { esquerdo) }\end{array}$ \\
\hline $\begin{array}{l}\text { Memória } \\
\text { de trabalho } \\
\text { verbal }\end{array}$ & $\begin{array}{l}\text { Internalizar } \\
\text { linguagem receptiva; } \\
\text { internalizar } \\
\text { linguagem } \\
\text { expressiva. }\end{array}$ & $\begin{array}{l}\text { Autodescrição, reflexão, } \\
\text { instrução e questionamento, } \\
\text { Comportamento governado } \\
\text { por regras; compreensão de } \\
\text { leitura; Controle moral da } \\
\text { conduta. }\end{array}$ & $\begin{array}{l}\text { Pré-frontal } \\
\text { dorsolateral } \\
\text { (esquerdo maior } \\
\text { direito) }\end{array}$ \\
\hline $\begin{array}{l}\text { Auto- } \\
\text { regulação } \\
\text { da emoção e } \\
\text { motivação }\end{array}$ & $\begin{array}{l}\text { Internalização de } \\
\text { expressões afetivas; } \\
\text { Intenalização } \\
\text { do estado de } \\
\text { motivação; } \\
\text { Internalização } \\
\text { da regulação da } \\
\text { motivação. }\end{array}$ & $\begin{array}{l}\text { Autocontrole da emoção; } \\
\text { motivação intrínseca/ } \\
\text { persistência; Ativação para } \\
\text { demanda das tarefas. }\end{array}$ & $\begin{array}{l}\text { Pré-frontal } \\
\text { ventromedial }\end{array}$ \\
\hline Reconstituição & Análise Síntese & $\begin{array}{l}\text { Fluência verbal/ não verbal, } \\
\text { criatividade dirigida ao } \\
\text { objetivo; flexibilidade e } \\
\text { sintaxe de idéias. }\end{array}$ & $\begin{array}{l}\text { Polo pré-frontal } \\
\text { anterior }\end{array}$ \\
\hline
\end{tabular}


Esse modelo concebe a memória de trabalho em termos de algumas características dos processos componentes, principalmente sua dinâmica temporal no cérebro. Uma dessas características é a eficiência de processamento, operacionalizada como velocidade de processamento. A velocidade de processamento tem sido postulada como fator de mediação entre o construto psicológico da inteligência fluida e a sua base biológica, (Carpenter, Just, \& Reichle, 2000) bem como do desenvolvimento do cognitivo ligado às funções executivas (Salthouse, 1994; Salthouse \& Babcock, 1991). Muitos pesquisadores consideram que a capacidade de sequenciamento de operações pode ser explicada em função da velocidade de processamento. Quanto mais rapidamente forem executadas as operações mentais, maior será a capacidade de armazenamento em um dado momento psicológico. Uma outra característica corresponde a um processo propriamente dito e consiste na capacidade de armazenamento temporário, dependente de descargas neuronais sustentadas em células distribuídas ao longo de uma rede de retroalimentação amplamente distribuída por regiões corticais e subcorticais (Fuster, 1995; Goldman-Rakic, 1996). Finalmente, a última característica consiste na capacidade de coordenação entre as diversas operações, principalmente coordenação entre armazenamento e execução de operações de solução de problemas, correspondendo ao aspecto verdadeiro "executivo" do modelo. Do ponto de vista neurobiológico existem diversas propostas sobre como isso poderia ocorrer a partir da organização do timing na atividade neuronal. Essas propostas variam desde o mecanismo de sincronização oscilatória proposta por Singer (Singer, 1998; Sarnthein, Petsche, Rappelsberger, Shaw, \& von Stein, 1998; Smith, \& Jonides, 1993) até o mecanismo de integração por oscilações periódicas proposto por Pöppel $(1994,1997)$.

O modelo de memória de trabalho pode oferecer grande atrativo, portanto, do ponto de vista da construção do construto psicológico.Por um lado, o modelo foi psicometricamente operacionalizado, como veremos a seguir, de um modo que permite seu mapeamento em uma das formas de armazenamento visual e auditiva, a inteligência fluida ou o raciocínio indutivo, justamente aquela forma de inteligência que se acredita ter uma maior fundamentação genético-biológica. Ao mesmo tempo, um dos conceitos fundamentais do modelo é representado pela velocidade de processamento, que, não por acaso, parece mediar às relações entre biologia e inteligência. Por outro lado, a natureza dos processos definidos permite correlacioná-los com dados empíricos a respeito do papel das descargas neuronais sustentadas na memória de trabalho. Permite também correlacioná-los com modelos mais recentes oriundos da dinâmica cerebral que procuram explicar o funcionamento executivo em termos de mecanismos neuronais ao nível celular.

Por outro lado será possível que, através da aplicação do teste e de sua análise, possamos tornar mais evidente os déficits comportamentais e 
as implicações das dificuldades no cotidiano escolar e do grupo estudado, sobretudo nos aspectos relativos às funções executivas e às habilidades menos dependentes da linguagem e seus possíveis distúrbios, que são as funções nas quais se observa a presença mais saliente do raciocínio indutivo e da memória de trabalho avaliadas aqui.

\section{Procedimentos metodológicos}

Apesar de ser o TDAH um dos transtornos mais frequentes na infância e consequentemente um dos mais estudados na população infantil, ainda são poucos os trabalhos que visam relacionar a função cognitiva da memória de trabalho com as áreas de dificuldade de aprendizagem no transtorno do déficit de atenção/hiperatividade, em crianças brasileiras, sendo esse o principal e maior objetivo desse projeto.

Portanto, esse estudo teve como objetivo investigar a memória de trabalho em crianças diagnosticadas com TDAH dos três subtipos: (a) predominantemente desatenta, (b) do tipo hiperativo/impulsivo e (c) do tipo combinado, bem como lançar luz de uma descrição na correlação entre os aspectos ligados às dificuldades em situação escolar (dificuldades relacionadas às áreas de matemática, de leitura e escrita).

Para realização desse trabalho, foi necessário verificar as dificuldades de aprendizagem no funcionamento escolar de cada criança, para a caracterização de um perfil de funcionamento dos mesmos no desempenho das tarefas de memória de trabalho, e por fim avaliar a capacidade armazenamento auditiva e visual e a velocidade de processamento no desempenho do instrumento.

Para a realização dessa pesquisa, o método escolhido é um estudo correlacional, onde as crianças com TDAH foram avaliadas uma única vez e comparadas com o grupo controle. Foram selecionados 62 sujeitos, sendo 32 sujeitos com diagnóstico de TDAH, de acordo com os critérios de avaliação do DSM-IV, 1994, meninos e meninas, com faixa etária entre 6 a 15 anos, que estejam frequentando escola. Esses 32 sujeitos com TDAH foram separados em três grupos com o número de 10 e 11 sujeitos para cada grupo, cada grupo apresentou diagnóstico de tríade sintomatológica do TDAH. Sendo 10 sujeitos do sexo feminino do tipo predominantemente desatento; 11 sujeitos de ambos os sexos do tipo hiperativo/ impulsivo e 11 sujeitos de ambos os sexos do tipo combinado.

Quanto aos sujeitos controles, foram selecionados 30 sujeitos normais, sendo 10 sujeitos do sexo feminino e 20 sujeitos de ambos os sexos, todos foram pareados com o grupo experimental.

Para a realização desta pesquisa, foi usado o Teste Infantil de Habilidade Cognitiva (TIHC), desenvolvido e adaptado por Primi (2002). Esse instrumento contém cinco conjuntos de tarefas ou subtestes que avaliam 
a memória de prazo curto e a inteligência: 1) Memória de Armazenamento Auditiva; 2) Memória de Armazenamento Visual; 3) Memória de Trabalho Auditiva; 4) Memória de Trabalho Visual; e 5) Raciocínio Indutivo.

A aplicação dos testes foi feita de forma individual em tempo médio de 30 minutos para cada examinando. Iniciou-se a aplicação com a tarefa de Memória de Armazenamento Visual, passando para a tarefa de Memória de Armazenamento Auditivo, após um intervalo de 5 minutos foi dada continuidade à tarefa de Memória de Trabalho Visual e, por fim, a tarefa de Memória de Trabalho Auditivo. Foi agendado um outro dia para a prova de Raciocínio Indutivo, o tempo estimado para essa prova foi de 10 a 30 minutos. Cabe salientar que as tarefas com de Memória de Trabalho Visual, Memória de Armazenamento Visual e o Raciocínio Indutivo são autoaplicáveis, cabendo ao examinador apenas as explicações.

A aplicação do teste foi feita de forma individual em tempo médio de 30 minutos para cada examinando. As crianças com TDAH foram levadas, individualmente, à sala de aplicação (ADHD-SEPIA-HC-IPq-USP) pelo examinador, onde foi realizado um breve rapport, com o objetivo de deixar a criança à vontade. Foi feito o mesmo procedimento para com as crianças normais, o teste foi aplicado em uma sala (sala de estudo) definida pela administração da escola em questão ventilada e sem ruídos.

\section{Considerações finais}

Esse trabalho investigou habilidades cognitivas da memória de crianças com o transtorno do déficit de atenção e hiperatividade, através de cinco subtestes informatizados. As medidas de raciocínio e memória fornecidas pelo Teste Infantil de Habilidades Cognitivas (TIHC) foram comparadas nos subgrupos de TDAH. Essa análise inicial foi executada com vistas a cumprir o objetivo determinado para esse estudo, de comparar a memória nos subgrupos de crianças com TDAH, ou seja, aquelas predominantemente desatentas, hiperativas/impulsivas e combinadas. Foi utilizada a prova One-Way Wilcoxon W, com nível de significância de 0,05.

De modo geral os instrumentos apresentam dados interessantes e aceitáveis quanto à caracterização do perfil neuropsicológico dos diferentes subtipos de TDAH. A organização foi dividida em quatro fatores interrelacionados, a saber, Raciocínio Indutivo, ligado à Inteligência, Extensão de Memória de Trabalho Auditiva e Visual ligados à Memória de Curto Prazo, capacidade de Armazenamento Auditiva e Visual ligados à Memória de Trabalho, e Tempo de Reação ligado ao fator Velocidade de Decisão. Os subtestes estão também correlacionados significativamente a variáveis externas independentes colhidas pelos professores, versando sobre dificuldade que os grupos de TDAH possuem, indicando evidências positivas de validade com referência ao critério. 
Estes últimos resultados associados à validade de critério foram explorados à luz das associações com o esquema de Pennington (1997) associado ao modelo $\mathrm{CHC}$ discutido na introdução. A função executiva que vem sendo associada à área pré-frontal do cérebro é responsável pela habilidade associada à memória de trabalho e funções correlatas como planejamento, organização, atenção seletiva, controle inibitório (Primi, 2002). Essa função é avaliada por tarefas ligadas à Memória de Armazenamento e de Trabalho e ao Raciocínio Indutivo. Distúrbios relacionados a essa capacidade teriam como resultado distúrbio do déficit de atenção. Já a cognição espacial, geralmente localizada no hemisfério direito posterior do cérebro, é responsável pelas habilidades como localização e identificação de objetos, memória visual ou espacial e outros elementos do processamento visual. Essa função está relacionada às habilidades cognitivas, envolvendo, principalmente, o processamento visual, o conhecimento quantitativo e o raciocínio indutivo. Distúrbios relacionados a essas capacidades teriam como resultado os distúrbios específicos nas áreas de cálculos e na escrita manual.

De um modo geral, as crianças com TDAH apresentaram um resultado inferior ao do grupo controle no desempenho, em quase todas as provas, exceto pela Memória de Trabalho Visual. Apesar de esse grupo obter escores menores não foi significativamente inferior aos do grupo das crianças controles, ou seja, ambos os grupos apresentaram medidas próximas na realização dos subtestes, enquanto que o tempo reação, de velocidade de processamento das informações auditivas e visuais, e tempo de execução foram significativamente menores para o grupo de TDAH.

Tendo obtido tais resultados conclui-se que uma menor capacidade no desempenho das tarefas de Memória de Trabalho e Armazenamento para o grupo TDAH esteve relacionada com um Tempo de Reação menor. Esses efeitos podem estar correlacionados às dificuldades decorrentes de maior esforço em manter a atenção. As crianças com esse transtorno apresentam maior desatenção e esta, provavelmente, seria uma das responsáveis pelo baixo desempenho.

Os componentes cognitivos necessários nas tarefas de Memória de Trabalho e Armazenamento e Raciocínio Indutivo envolvem a tomada de decisões, o planejamento de estratégias e o controle da atenção por meio da integração das informações dos sistemas subordinados (buffers) da memória de trabalho. Além desses recursos cognitivos, demanda a capacidade de abstração do sujeito na tarefa de Raciocínio Indutivo. A abstração está intimamente ligada ao controle de atenção, estando a dificuldade nessas capacidades ligadas diretamente aos déficits encontrados no grupo de TDAH. Esses recursos cognitivos envolvem associações corticais difusas, que requerem comunicações da memória de curto prazo, memória de trabalho e memória de longo prazo. Portanto, a dinâmica cortical pode 
ultrapassar/sobrecarregar a capacidade de memória de trabalho dessas crianças.

Foram observadas similaridades na capacidade mental do grupo TDAH e no grupo controle na tarefa de Raciocínio Indutivo. Esse fato apresentou um dado interessante, pois, apesar do grupo de TDAH ser mais rápido que o grupo controle, estes não cometeram tantos erros na tarefa. $O$ que pode ter ocorrido é que o grupo de TDAH pode ter usado mais seu processo atencional que compreende uma estratégia de construção de áreas deficitárias nesse transtorno, com ajuda de outros componentes positivos do transtorno virão em "socorro" dos componentes problemáticos nessas crianças.

O tempo de reação do TDAH foi um dado significativo em todo o teste, podendo-se intuir que as configurações dos déficits nesses indivíduos tiveram uma participação decisiva nas respostas dadas. $O$ processo desinibido da morfologia do TDAH (hiperatividade e impulsividade) torna plausível respostas mais rápidas e menos "freios" no comportamento desses sujeitos.

Um outro dado interessante foi colhido nas provas de Memória de Armazenamento Auditiva e Memória de Trabalho Auditiva. Essas provas envolvem recursos dos sistemas subordinados da alça fonológica, sendo um destinado à estocagem fonológica passiva, outro relativo aos processos de ensaio articulatórios que apresentam capacidade limitada (decai entre 1 a 2 segundos se não ensaiados através de repetição subvocal). Esses recursos são responsáveis pela manipulação e armazenamento do material verbal. Para isso, a reprodução das palavras nessa tarefa dependia da estocagem passiva sem tempo para agregar significado.

O fato de o grupo de TDAH ter denotado médias próximas ao grupo controle, deve-se aos efeitos de similaridade fonológica das palavras (bicho, pedra, homem, peixe) no teste, ou seja, palavras que tenham acesso ao significado e que façam parte do seu vocabulário, tendo a pouca extensão da palavra (palavras curtas) também ajudada nessa recordação. Além disso, apresentaram-se palavras monossílabas e dissílabas nessa prova, não tendo a criança com esse transtorno conseguido passar para próxima configuração com três itens contendo palavras trissílabas, ou seja, o grupo de TDAH conseguiu recordar somente oito palavras no total, sendo 6 palavras da extensão de 2 itens e 2 palavras da extensão de 3 itens. Esse grupo apresentou maior dificuldade para recuperar informações verbais, apresentando a extensão de 6 palavras, sendo que não conseguiram chegar à segunda extensão com três itens. Nessa condição, o grupo controle teve maior facilidade para recuperar a informação verbal, apresentando 11 palavras recordadas, embora não tenham ultrapassado a segunda extensão com 3 itens.

Pôde-se estabelecer um limite de palavras nessa tarefa para as crianças com TDAH, tendo sido 7 o limite de palavras para o subtipo desatento e 
6 para os subtipos hiperativos e combinado. De fato, para sete palavras nenhuma criança do grupo conseguiu realizar toda a tarefa, demonstrando assim que a capacidade de armazenamento, para a faixa etária estudada, foi ultrapassada. Isso se deve à desatenção presente nessas crianças, fazendo com que as mesmas não utilizem o recurso de associação semântica tão eficazmente quanto as crianças controle, de forma a facilitar a recordação do conteúdo verbal. Pode ser observado que além desses recursos do grupo controle, diferente do grupo TDAH, esse primeiro procurava repetir internamente as palavras durante a aplicação, enquanto que o grupo TDAH não apresentou estratégia para recuperação do material verbal. Ainda foi observado, durante a prova, que esse subgrupo antecipava muito as respostas prejudicando o recebimento dos itens seguintes. Esse dado pôde ser colhido porque a tarefa foi apresentada pelo examinador, enquanto a criança tinha que repetir a sequência de palavras ouvidas no áudio do computador e o examinador "clicava" com o mouse na tela do computador as respostas dadas pelas crianças.

Esses resultados estão de acordo com a literatura, pois a limitação de tempo em que a informação pode ser mantida determina ainda que palavras mais curtas tendam a ser mais bem lembradas, já que são pronunciadas mais rapidamente (Baddeley, Thomson, \& Buchanan, 1975). Esse sistema funciona através de código fonológico (Anderson et al., 2000), assim, itens com sonoridade similar, que possuem códigos de estocagem capazes de se confundir, são mais vulneráveis aos efeitos do esquecimento.

Análogo à Memória de Armazenamento e de Trabalho Auditiva, a Memória de Armazenamento e de Trabalho Visual também recruta sistemas subordinados, um responsável pela manutenção passiva de material visual, outro pela manipulação de material espacial.

No que se refere à Memória de Trabalho Visual, o grupo de TDAH apresentou maior capacidade de recuperação e execução nessa tarefa. $O$ tempo gasto pelo grupo de TDAH foi menor que o grupo controle, mas, ainda assim, denotou maior capacidade mental nessa habilidade.

Esses dados são interessantes, uma vez que a Memória de Trabalho Visual depende dos componentes subordinados da alça vísuo-espacial (estocagem passiva e articulações), utilizando um conjunto de exigências para reaver o material visual-espacial que depende de operação da memória de trabalho e do central executivo. É possível inferir, então, que as crianças com TDAH, apesar da melhora na percepção e atenção, continuarão apresentando dificuldades no aprendizado das áreas que necessitam da Memória de Trabalho Auditiva e dos conceitos verbais para seu desenvolvimento, o que não acontece para Memória de Trabalho Visual.

Por outro lado, não é possível dizer que nas crianças com esse transtorno existam diferenças no desempenho da Memória de Trabalho Visual (MTV) em comparação com a Memória de Trabalho Auditiva (MTA), uma vez que as crianças com TDAH mostraram dificuldades em provas relacio- 
nadas a ambas as memórias. Portanto, as conclusões desse trabalho não confirmaram a hipótese de que as crianças com TDAH apresentariam um menor desempenho na Memória Visual do que na Auditiva.

Em suma, observa-se que as crianças com TDAH beneficiam-se menos dos recursos verbais e mais dos visuais. Isso pode ter consequências para a aprendizagem da leitura e um menor aproveitamento das informações do ambiente. Essas crianças parecem estabelecer menos associações e aproveitam mal os recursos semânticos e fonológicos contidos nas palavras e, consequentemente, o desempenho é prejudicado pela dificuldade que apresentam para integrar as informações.

É provável que a baixa capacidade na memória de trabalho esteja relacionada a um baixo desempenho atencional, que também pôde ser observado nesse estudo. Essa desatenção levaria a uma utilização menor dos recursos mentais, causando, assim, um prejuízo na retenção de informações de memória de curto prazo.

Os resultados obtidos nos questionários de dificuldades de aprendizagem constataram que as crianças com TDAH não tiveram maiores dificuldades de aprendizagem em quaisquer das áreas estudadas, de modo geral.

O TDAH é, então, um tipo de distúrbio de aprendizagem. Pode ser acompanhado de outros transtornos, como dislexia ou distúrbio de memória, ou por uma dificuldade específica de aprendizagem, como a relacionada à matemática (discalculia). De início, essa distinção entre dificuldade específica e distúrbio propriamente dito pode causar confusão. As dificuldades específicas (ou distúrbios específicos) são umas subdivisões dos distúrbios e afetam aptidões bem definidas: dificuldade específica em matemática, linguagem, ortografia. Os distúrbios, por outro lado, não são tão definidos, afetando a cognição em geral.

Uma vez que o TDAH afeta todas as áreas da cognição, tende a exacerbar qualquer dificuldade específica de aprendizagem. O TDAH não é um distúrbio específico de aprendizagem, pois não inviabiliza qualquer função cognitiva, é mais amplo que isso. Uma dificuldade específica em matemática pode ser comumente encontrada com o TDAH, assim como outra dificuldade específica, especialmente a de aprender idiomas.

Entretanto, sabe-se que a memória de trabalho é expressa nas áreas frontais do cérebro e, sendo as áreas frontais das crianças com TDAH, hipoativas, pode-se concluir que as crianças com TDAH têm a memória de trabalho prejudicada? Provavelmente, não de forma conclusiva. Ainda assim, talvez as pesquisas futuras apontem isso, à medida que os métodos forem tornando-se mais sofisticados.

Em suma, na relação entre a memória de trabalho e o TDAH, no que se refere aos aspectos encontrados nesse trabalho, investigou-se o Raciocínio Indutivo, a Memória de Trabalho e Armazenamento de informações verbais e visuais, com a identificação de quais processos podem estar por 
trás do processamento dessas informações. Os resultados colhidos neste trabalho demonstram que muitas vezes vêm-se atribuídos rótulos a essas crianças erroneamente, já que a capacidade de raciocínio indutivo/inteligência não demonstrou aqui ser inferior para essas crianças em relação às crianças normais. Além disso, verificou-se, nesse estudo, que as crianças com TDAH apresentaram características de capacidades cognitivas e de aprendizagem próximas às crianças normais. Espera-se que esse trabaIho possa ajudar a compreender melhor as crianças e adolescentes com "Transtorno do Déficit de Atenção e Hiperatividade".

\title{
Assessment of working memory in children with attention deficit hyperactivity disorder
}

\begin{abstract}
This research investigated the cognitive abilities and the Working Memory in children and youngsters with Attention-Deficit/Hyperactivity Disorder (ADHD). The results of a computerized test called Infant Cognitive Abilities Test (TIHC), that measures five cognitive abilities (1- inductive reasoning (IR), 2- auditory short-term memory (AM), 3- visual short-term memory (VM), 4- auditory working memory (AWM) and 5- visual working memory (VWM)) based on the Cattell-HornCarroll model. Thirty-two children from both sexes, aged between 7 and 15 years, from the Hospital das Clínicas-SEPIA-USP, suffering from ADHD and a control group of thirty children from a State Elementary School were submitted to the test. The results showed that there are significant differences regarding the working memory of the different types of ADHD. Children suffering from ADHD had high test scores for the visual memory items of the test when compared to the results in the auditory memory items. In the computerized TIHC the reaction time of the short-term visual memory test showed a significant correlation with the mathematic test variable.
\end{abstract}

Keywords: Memory. Attention-deficit. Hyperactivity. ADHD. Neuropsychology.

\section{Évaluation de la mémoire de travail dans des enfants avec bouleversement du déficit d'attention et hiperatividade}

Résumé: Ce projet a enquêté aux habilités plus cognitives de la Mémoire de Travail d'enfants et d'adolescents avec le «Bouleversement du Déficit Atenção et de Hyperactivité » (TDAH). L'essai TIHC-Teste Infantile à d'Habilités Plus cognitives informatisé a été apliqué, en évaluant principalement 5 habilités les plus cognitives Raisonnement Inductives (IL RIT), Mémoire de Stockage Auditif (MAA) et Aspect et Mémoire de Travail Auditive (MTA) et Aspect (MTV) en utilisant le modèle 
de Cattell-Horn-Carroll. L'essai a été appliqué dans 62 sujets, en étant 32 avec TDAH de l'Hôpital du Clínicas-SEPIA-USP et 30 sujets contrôle " normal » de l'enseignement

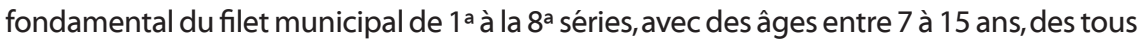
les deux sexes. Les analyses exécutées ont indiqué l'existence de différences dans de divers aspects rapportés à la mémoire dans les types particuliers d'enfants avec TDAH.II s'est vérifié que les enfants avec TDAH ont obtenu bonne performance dans les preuves de mémoire visuelle dans détriment les preuves de mémoire auditive. La corrélation des résultats de TIHC s'est vérifiée que le temps de réaction de la preuve de mémoire de stockage visuel a maintenu une corrélation significative avec la preuve de calcul.

Mots-clés: Mémoire. Déficit d'attention. Hyperactivité.TDAH. Neuropsychologie

\section{Evaluación de la memoria de trabajo en niños con transtorno de déficit de atención e hiperctividad}

Resumen: Ese proyecto investigó las capacidades cognitivas y la memoria de trabajo en niños y jóvenes con transtorno de déficit de atención e hiperctividad (TDAH). Se aplicó el test TIHC-Teste Infantil de Habilidades Cognitivas informatizado, evaluando principalmente cinco capacidades cognitivas: el razonamiento inductivo (RI), memoria a corto plazo auditiva y visual y memoria de funcionamiento auditiva y visual de acuerdo con el modelo de Cattell-Cuerno-Carroll. Sesenta y dos personas hicieron el test. Eran treinte y dos con TDAH del Hospital das Clínicas-SEPIA-USP y treinta sujetos control "normal"de la enseñanza básica de escuelas públicas de 1 ro a 8vo año con edades entre 7y 15 años, de ambos los sexos. Las análisis demostraron que hay diferencias significativas con respecto a la memoria de trabajo de los diversos tipos de TDAH. Los niños que sufrían de TDAH tenían altas puntuaciones del test para los artículos visuales de la memoria cuando comparados a los resultados en los artículos auditivos de la memoria. En el TIHC se verificó que el tiempo de reacción de la prueba de memoria a corto plazo visual mantuvo una correlación significativa con la prueba de cálculo.

Palabras-clave: Memoria. Déficit de atención. Hiperactividad.TDAH. Neuropsicología.

\section{Referências}

Adams, A. A., \& Gathercole, S. E. (1996). Phonological working memory and spoken language development in young children. The Quarterly journal of Experimental Psychology, 49(1), 216-233. 
American Psychiatry Association. (1994). Diagnostic and statistical manual of mental disorders (4th ed.). Washington, DC: Author.

Anderson, G. M., Dover, M. A., Yang, B. P., Holahan, J. M., Shaywitz, S. E., Marchione, K. E., et al. (2000). Adrenomedullary function during cognitive testing in attentiondeficit/hyperactivity disorder. Journal of American of Academy of Child and Adolescence Psychiatry, 39(5), 635-643.

Arnold, L. E., Abikoff, H. B., Cantwell, D. P., Conners, C. K., Elliott, G.; Greenhill, L. L., et al. (1997). National Institute of Mental Health Collaborative Multimodal Treatment Study of Children with ADHD (the MTA). Design challenges and choices. Archives of General Psychiatry, 54(9), 865-870.

Babinski, L. M., Hartsough, C. S., \& Lambert, N. M. (1999). Childhood conduct problems, hypreractivity-impulsivity, and inattention as predictions of adult criminal activity. Journal of Child Psychology and Psychiatry, 40(3), 347-355.

Baddeley, A. (1996a). Exploring the central executive. The Quarterly Journal of Experimental Psychology, 49(1), 5-28.

Baddeley, A. (1996b). The fractionation of working memory. Proceedings of the National Academy of Sciences (USA), 93, 13468-13472.

Baddeley, A. (1998). Human memory: Theory and practice. London: Allyn \& Bacon.

Baddeley, A., Thomson, N., \& Buchanan, M. (1975). Word Length and the structure of short-term memory. Journal of Verbal Learning and Verbal Behavior, 14, 123-144.

Balthazor, M. J, Wagner, R. K, \& Pehan, W. E. (1991). The specific of the effects of stimulate medication on classic learning related measures of cognitive processing for attention deficit disorders children. Journal of Abnormal Child Psychology, 19(1), 35-52.

Barbosa, G. A., Barbosa, A. A. G., \& Nascimento, J. A. (1999a). Existe correlação entre transtorno hipercinético e sinais neurológicos menores? Um estudo com a escala Paness, Infanto: Revista de Neuropsiquiatria da Infância e Adolescência, 7(1), 39-46.

Barbosa, G. A., Barbosa, A. A.G., \& Nascimento, J. A. (1999b). Uso do P.A.C.S. em crianças hipercinéticas. Infanto: Revista de Neuropsiquiatria da Infância e Adolescência, 7(3), 97-105.

Barbosa, G. A., Gouveia, V. V., \& Barbosa, A. A. G. (1993). O Fator Hiperatividade do Questionário de Conners: validade conceitual e normas diagnosticas. Temas, 46, 188-202.

Barbosa, G. A. Gouveia, V. V, \& Barbosa, A. A. G. (2000). Fatores predisponentes da síndrome hipercinética: um estudo retrospectivo. Infanto: Revista de Neuropsiquiatria da Infância e Adolescência, 8(2), 73-80.

Barkley, R. A. (1996). Cognitive behavioral therapy with ADHD children. New York: The Guilford.

Barkley, R. A. (1997). Behavioral inhibition, sustained attention, and executive functions: Constructing a unifying theory of ADHD. Psychology Bulletin, 121, 65-94.

Barkley, R. A. (2000). Genetics childhood disorders: XVII. ADHD, Part 1: The executive functions and ADHD. Journal of American of Academy of Child and Adolescence Psychiatry, 39(8), 1064-1067. 
Barkley, R. A., Fisher, M., Newby, R. F., \& Breen, M. J. (1988). Development a multimethod clinical protocol for assessing stimulant drug response in children with attention deficit disorder. Journal of Clinical Child Psychology, 17, 14-24.

Barkley, R. A., Grodzinsky, G., \& DuPaul, G. J. (1992). Frontal lobe functions in attention deficit disorder with and without hyperactivity: A review and research report. Journal of Abnormal Child Psychology, 20,163-88.

Carpenter, M. B. (1978). Neuroanatomia humana interamericana (7a ed.). Porto Alegre: Artes Médicas.

Carpenter, P. A. Just, M. A., \& Shell, P. (1990). What one intelligence test measures: A theoretical account of the processing in the Raven progressive Matrices test. Psychological Review, 97(3), 404-431.

Carpenter, P. A., Just, M. A., \& Reichle, E. D. (2000). Working memory and executive function: Evidence from neuroimaging. Current Opinion in Neurobiology, 10, 1951990.

Castellanos, F. X. (1997). Toward a path physiology of attention-deficit/hyperactivity disorder. Clinical Pediatrics, 36, 381-393

Castellanos, F. X., Giedd, J. N., Ellia, J., Marsh, W. L. Ritchie, G. F., Hamburger, S. D., et al. (1997). Controlled stimulant treatment of ADHD and comorbid Tourettes' syndrome: Effects of stimulant and dose. Journal of American of Academy of Child and Adolescence Psychiatry, 36(5), 589-596.

Castellanos, F. X., Ducharme, F. F. M. J. L., Walter, J. M., Israel, M. E., Krain, A., \& Pavlovsky, C., (2000). Executive function oculomotor tasks in girls with ADHD. Journal of American of Academy of Child and Adolescence Psychiatry, 39(5), 644-650.

Cohen, N. J., Vallance, D. D., Barwick, M. I. M. N., Menna, R., Horodezky, N. B., \& Issacson, L. (2000). The interface between ADHD and language impairment: An examination of language, achievement, and cognitive processing. Journal of Child Psychology and Psychiatry, 41(3), 353-362.

Carroll, J. B. (1993). Human cognitive abilities: A survey of factor-analytic studies. New York: Cambridge University Press.

Del Nero, H. S. (1997). O sítio da mente: pensamento, emoção e vontade no cérebro humano. São Paulo: Collegium Cognition.

Farner, C. M., \& O’Donnell, B. F. (2000). Visual perception memory in schizotypal personality disorder. American Journal Psychiatry, 157(5), 781-788.

Fuster, J. M. (1995). Memory in the cerebral cortex. An empirical approach to neural networks in the human and nonhuman primate. Cambridge, MA: MIT Press.

Gathercole, S. E., \& Baddeley, A. D. (1993). Working memory and language. Hillsdale, $\mathrm{NJ}$ : Lawrence Erbraum.

Goldman-Rakic, P. S. (1996). Regional and cellular fractionation of working memory. Proceedings of the National Academy of Sciences (USA), 93, 13473-13480.

Kendall, P. C, \& Chansky, T. A. (1991). Considering cognition in anxiety disordered children. Journal of Anxiety Disorders, 5, 167-185.

Kuntsi, J., Oosterlaan, J., \& Stevenson, J. (2001). Psychological mechanisms in 
hyperactivity: I response inhibition deficit, working memory impairment, delay aversion, or something else? Journal of Child Psychology, 42(2), 199-210.

Meili, R. (1985). The effect of environmental stimulation upon social and exploratory behavior in human infant. In B. M. Foss (Ed.), Determinants of infant behavior ( $\mathrm{p}$. 10-59). New York: Wiley Press.

Pasquali, L. (2001). Técnicas e Exames Psicológicos - TEP: Vol. I - Fundamentos das Técnicas de Exames Psicológicos. Brasília, DF: Casa do Psicólogo.

Pennington, B. F. (1997). Using genetics to dissect cognition. American Journal Human Genetics, 60, 13-16.

Pennington, B. F., \& Ozonoff, S. (1996). Executive functions and developmental psychopathology. Journal of Child Psychology and Psychiatry, 37(1), 51-87.

Pöppel, E. (1994). Temporal mechanisms in perception. International. Review of Neurobiology, 37, 185-202.

Pöppel, E. (1997). Antidepressants in the treatment of attention-deficit/hyperactivity disorder. Journal Clinical. Psychiatry, 58(Supl. 14),14-29.

Posner, M. I., \& Cohen, Y. (1984). Components of visual orienting. In H. Bouwma \& D. G. Bowhuis (Ed.), Attention and performance X (pp. 130-146). Hillsdale, NJ: Lawrence Erlbaum.

Primi, R. (2002). Complexity of geometric inductive reasoning tasks: Contribution to the understandings of the fluid intelligence. Intelligence, 30(1), 41-70.

Rohde, L. A., Biederman, J., Knijnik, M. P., Ketzer, C., Chachamovich, E., Vieira, G. M. et al. (1998). Exploring DSM-IV ADHD number of symptoms criterion: Preliminary findings in adolescents. Infanto: Revista de Neuropsiquiatria da Infância e Adolescência, 6(3), 114-118.

Rohde, L. A. (1997). Tratamento de déficit de atenção/hiperatividade: um estudo de prevalência, co-morbidade, fatores associados e critérios diagnósticos em escolares de 12-14 anos da rede estadual de Porto Alegre. Tese de Doutorado, Faculdade de Medicina da Universidade Federal do Rio Grande do Sul, Porto Alegre.

Salthouse, T. A. (1994). The aging of working memory. Neuropsychogy, 8(4), 535-543.

Salthouse, T. A., \& Babcock, R. L (1991). Decomposing adult age differences in working memory. Developmental psychology, 27(5), 763-776.

Sarnthein, J., Petsche, H., Rappelsberger, P., Shaw, G. L., \& von Stein, A. (1998). Synchronization between prefrontal and posterior association cortex during human working memory. Proceedings of the National Academy of Sciences (USA), 95, 7092-7096.

Singer, W. (1998). Consciousness and the structure of neuronal representations. Philosophical Transactions of the Royal Society of London B, 353, 1829-1840.

Smith, E. E., \& Jonides, J. (1998). Neuroimaging analyses of human working memory. Proceedings of the National Academy of Sciences (USA), 95, 12061-12068.

Sonuga-Barke, E. J, Taylor, E., \& Sembi, S. (1992). Hyperactivity and delay aversion-I. The effect of delay on choice. Journal of Child Psychology and Psychiatry, 33, 387-98. 
Strauss, M. E., \& Alphal Boekamp J. (1992). Disengagement of attention in chronic schizophrenia and hyperactivity child. Psychiatric Research, 43(1), 87-92.

Swanson, J., Deutsch, C., Cantwell, D., Posner, M., Kennedy, J. L., \& Barr, C. L. (2001). Genes and attention-deficit/hyperactivity disorder. Clinical Neuroscientist Resume, 1, 207-216.

Swanson, J. M., \& Posner, M. I. (1991). Activating task for the study of visual-spatial attention in ADHD children: A cognitive anatomic approach. Journal Child Neuropsychology, 6(1), 119-127.

Swanson, J. M., Sunohara, G. A., Kennedy, J. L., Regino, R., Fineberg, E., \& Wigal, T., (1998). Association of the dopamine receptor D4 (DRD4) gene with a refined phenotype of attention-deficit/hyperactivity disorder (ADHD): A family-based approach. Molecule Psychiatry, 3, 38-41.

Swanson, J., Posner, M. I., Cantwell, D., Wigal, S., Crinella, F., Filipek, P., et al. (1998). Attention-deficit/hyperactivity disorder: Symptom domains, cognitive process, and neural networks. In R. Parasuraman (Ed.), The attentive brain (pp. 455-498). Massachusetts: A Bradford Book.

Teixeira, J. F. (1998). Mentes e máquinas: uma introdução à ciência cognitiva. Porto Alegre: Artes Médicas.

Watson, J. S., \& Ramey, C. T. (1978). Reactions to response-contingent stimulation in early infancy. Merrill-Palmer Quarterly, 18, 219-228.

Lucinete de Freitas Messina, Psicóloga da Florida International University-USA, Doutoranda em Psicologia do Núcleo de Pesquisa em Neurociência e Comportamento da Universidade de São Paulo. Endereço para correspondência: Av. Independência, 1699, CEP 13419155, Piracicaba, SP, Brasil.Endereço eletrônico: Ifremess@usp.br

Klaus Bruno Tiedemann, Professor do Departamento de Psicologia Experimental do Instituto de Psicologia da Universidade de São Paulo. Endereço para correspondência: Av.Prof. Mello Moraes, 1721,CEP 05508-030, Cidade Universitária, São Paulo. Endereço eletrônico: kbtiedemann@terra.com.br

Colaboradores/agradecimentos: IMIP - Instituto de Medicina Infantil de Piracicaba. Ananias, A. F.- Medico Neonatal do Instituto de Medicina Infantil de Piracicaba. Ferracciu, S. R. - Medica Imunologista do Inastituto de Medicina Infantil de Piracicaba. Giovelli I. J. - Medico Pediatrico do Instituo de Medicina de Piracicaba. Macruz Ferreira da Silva, O. J. - Medico Clinico Geral e Pediatra.

Recebido em: 2/01/2008

Aceito em: 25/06/2008 